\title{
One hundred and ten sports related facial fractures
}

\author{
Sean M Carroll, Mohamed A Jawad, Mark West and T P F O'Connor \\ Department of Plastic Surgery, Regional Hospital, Cork, Ireland
}

In a 1 year period (1993) 332 facial fractures were treated in Cork Regional Hospital. Of these $110(33 \%)$ were sports related. Males accounted for $94.5 \%$ of patients and $77 \%$ of patients were aged below 30 years. Nasal fractures were the most common fractures seen (56\%). Almost half (49\%) of sports related facial fractures occurred while playing Gaelic games. The incidence of hurling fractures has been more than halved over the last 20 years. Seventy eight per cent of patients required operative treatment.

(Br J Sports Med 1995; 27 : 194-195)

Keywords: facial fractures; sports; Gaelic games; hurling

Cork Regional Hospital is a tertiary referral centre servicing a population of 850000 . It is the regional trauma centre and has all specialties on site apart from ENT. The plastic surgery unit is the only one in the region and with the department of oral surgery exclusively treats all facial fractures occurring in the region, apart from those nasal fractures treated in the regional ENT unit. The oral surgeons treat the mandibular fractures and in conjunction with the plastic surgeons the Le Fort fractures. The plastic surgeons treat all other facial fractures, including all orbital blowout fractures. The experience of this unit therefore closely reflects the actual incidence of facial fractures in this region.

O'Donoghue et al ${ }^{12}$ reported from this unit in 1979 that sports related facial fractures accounted for $37 \%$ of all facial fractures treated. This figure is more than double that reported in the few studies that specifically address this subject. ${ }^{3-5}$ The aims of this study were therefore to establish what percentage of facial fractures are sports related, to perform an analysis of the aetiology of sports related facial fractures, to compare these data with similar data available from $1975,{ }^{12}$ and to suggest methods by which sports related facial fractures might be reduced.

\section{Methods}

The clinical notes of all patients with facial fractures seen in Cork Regional Hospital from the beginning of January to the end of December 1993 were reviewed. Only patients who sustained their fractures playing sports were included in this study. Data relating to age, sex, causative sport, fracture type, and treatment

Address for correspondence: Sean M Carroll FRCSI, Registrar in Plastic Surgery, Plastic Surgery Department, Cork Regional Hospital, Cork, Ireland. required were collected, stored, and analysed on a computer database. Relevant information was compared to previous publications from this unit on the aetiology of facial fractures in $1975 .^{12}$

\section{Results}

Of 332 patients seen with facial fractures over a 1 year period (1993), 110 (33\%) were sports related. This compares to a figure of $36.8 \%$ in 1975 .

\section{Age and sex}

Patients' age ranged from 9 to 67 years. Seventy per cent of patients were aged under 30 years. There was no difference in age profile between the causative sports. Males accounted for $94.5 \%$ of the patients $(n=104)$.

\section{Causative sport}

The Gaelic sports of football (29), hurling (23), and camogie (2) accounted for almost half of sports related facial fractures. Soccer (26) and rugby (14) were the next most common causes. Seven other sports caused the remaining 16 fractures: basketball, swimming, rock climbing, golf, hockey, martial arts, and horse riding. In 1975 hurling injuries comprised $20 \%$ of all fractures treated and $56 \%$ of sports related fractures. ${ }^{2}$ In 1993 hurling was the causative sport in $8 \%$ of the total and $21 \%$ of sports related fractures.

Soccer caused only $5 \%$ of sports related injuries in $1975^{2}$ but $23 \%$ in 1993 . The other main sports had similar fracture rates for the two periods (Table 1).

\section{Fracture type}

Of all sports related facial fractures, nasal fractures $(56 \%)$ occurred more often than zygomatic/maxillary (ZM) fractures $(23 \%)$ or mandibular fractures $(13 \%)$.

Table 1. Distribution and aetiology of facial fractures

\begin{tabular}{lrr}
\hline & 1975 & 1993 \\
\hline All causes & 266 & 332 \\
Sport & 98 & 110 \\
Hurling & 55 & 23 \\
Soccer & 5 & 26 \\
Rugby & 10 & 14 \\
Gaelic football & 22 & 29 \\
Other sports & 6 & 28 \\
\hline
\end{tabular}


There were four orbital floor fractures, four multiple facial fractures, and one Le Fort fracture. Nasal fractures were the most common fracture in every sport except soccer, where ZM fractures were more common. Only one $\mathrm{ZM}$ fracture occurred during hurling and none during rugby. In contrast, $46 \%$ of soccer injuries were $\mathrm{ZM}$ fractures (12 in all). The Le Fort fracture occurred during a climbing accident.

Overall, sport was the most common cause of nasal fracture, accounting for $35 \%$ of the total seen $(n=177)$. Sport was the second commonest cause of ZM fractures $(27 \%)$ and mandibular fractures $(24 \%)$, with assaults being the most common cause in both.

\section{Treatment}

Surgery was required in $78 \%$ of patients $(n=86)$. Fifty five per cent of operations were simple manipulations under anaesthetic of nasal fractures and $24 \%$ were Gillies elevations of zygomatic fractures. Maxillomandibular fixation was performed in seven patients, and a further seven patients required open reduction and internal fixation. Three of the four orbital fractures required exploration.

\section{Discussion}

We have noted that sports related facial fractures accounted for $33 \%$ of all facial fractures for this region in 1993. A similar figure (37\%) was noted by O'Donoghue for the same region in $1975 .{ }^{1}$ This contrasts sharply with studies from other units. A figure of $16.3 \%$ is reported from Australia, ${ }^{3} 9 \%$ from the USA, ${ }^{4}$ and $17.4 \%$ from Sweden. ${ }^{5}$ This difference may reflect local factors, such as a dilution of sports related facial fractures by other more common causes in these countries, or a higher level of participation in contact sports in this region. Alternatively, the differences may just reflect the varying proportions of facial fractures treated in these units. Unlike most other units, we are the only one in our hospital treating facial fractures. We therefore treat all the nasal fractures seen in the hospital. In fact we found nasal fractures to be the most common sports related facial fracture. Furthermore we found sport to have accounted for more nasal fractures than any other cause. This is in contrast to other papers on this subject which report that nasal fractures were among the least common sports related facial fracture seen by them. ${ }^{3} 6$ These authors point out that up to three different departments treat nasal fractures in their hospitals, leading to a likely underestimation of nasal fractures. ${ }^{36}$
As there is no such underestimation in our hospital, we therefore treat proportionately more nasal fractures than elsewhere, thus increasing the incidence of sports related facial fractures in our study.

Between 1975 and 1993 soccer related facial fractures increased nearly fivefold. This coincides with an increase in the number of soccer players in the region (secretary of the Irish FA, personal communication). It is of interest that zygomatic/maxillary fractures were more common in soccer than in any other sport. ZM fractures may be caused by heads clashing while heading the ball in soccer, but the use of the raised elbow to intimidate opponents undoubtedly also causes these fractures. As we have no specific information on the fracture causing incident, we cannot comment further but it is interesting to note that FIFA (the international association football federation) is considering banning the raising of the elbow above shoulder level in order to reduce this type of injury (secretary of the Irish FA, personal communication).

Between 1975 and 1993 the incidence of hurling related facial fractures was reduced by more than half. The nature of this game has been explained in a previous paper. ${ }^{7}$ The numbers of hurlers have increased during this time by about $50 \%$, but the introduction of head and face guards and improved coaching is thought to account for the game's improved safety (unpublished data). These modifications have not reduced the excitement of the game and show that improving the safety of a sport need not diminish its spectacle.

In conclusion, we have shown that over an 18 year gap the incidence of sports related facial fractures has remained consistently high at about one third of all facial fractures seen.

\section{References}

1 O'Donoghue GM, Vaughan EDV, Condon KC. An analysis of the pattern of facial injuries in a general accident department. Injury 1979; 11: 52-61.

2 O'Donoghue GM, Condon KC. Facial injuries in hurlers. J Irish Med Assoc 1979; 72 : 448-9.

3 Lim LH, Moore MH, Trott JA, David DJ. Sports-related facial fractures: a review of 137 patients. Aust N Z J Surg 1993; 63: 784-9.

4 Vetter JD, Topazian RG, Goldberg MH, Smith DG. Facial fractures occurring in a medium size metropolitan area: recent trends. Int $J$ Oral Maxillofac Surg 1991; 20: 214-6.

5 Starkhammer H, Ollofson J. Facial fractures: a review of 922 cases with special reference to incidence and aetiology. Clin Otolaryngol 1982; 7: 405-9.

6 Hill CM, Crosher RF, Mason DA. Dental and facial injuries following sports accidents: a study of 130 patients. $\mathrm{Br} \mathrm{J}$ Oral Maxillofac Surg $1985 ; 23$ : 268-74.

7 Crowley PJ, Condon KC. Analysis of hurling and camogie injuries. Br J Sports Med 1989; 23 : 183-5. 